\title{
General Topology of the Universe
}

\author{
Aalok Pandya ${ }^{1,2}$ \\ ${ }^{1}$ Department of Physics, University of Rajasthan, Jaipur, India \\ ${ }^{2}$ Department of Physics, JECRC University, Jaipur, India \\ Email: aalok.pandya@jecrcu.edu.in
}

Received 8 July 2014; revised 10 August 2014; accepted 20 August 2014

Copyright (C) 2014 by author and Scientific Research Publishing Inc.

This work is licensed under the Creative Commons Attribution International License (CC BY). http://creativecommons.org/licenses/by/4.0/

(c) (i) D Den Access

\begin{abstract}
General topology of the universe is described. It is concluded that topology of the present universe is greater or stronger than the topology of the universe in the past and topology of the future universe will be stronger or greater than the present topology of the universe. Consequently, the universe remains unbounded. The general topological approach comprises of powerful techniques that could prove to be useful to prescribe mathematical constraints on the global character of the universe as well as on the manifold of space-time.
\end{abstract}

\section{Keywords}

\section{General Topology, Manifold, Boundary, Causality, Euclidean Space, Discreteness, Limit Points}

\section{Introduction}

General topology of the universe and its evolutionary aspects are discussed in this paper. In the scenario of universe evolving with time, we conclude that topology of the present universe is greater or stronger than the topology of the universe in the past and topology of the future universe will be stronger or greater than the present topology of the universe. Consequently, the universe remains unbounded. The conditions of whole space topology can be treated as mathematical constraints on the global topology of the universe. The present exercise draws motivation from the recent explorations [1] [2] of relativistic aspects of the causal evolution of world lines and the earlier work on causal set topology by Joshi [3]. The Null-Cone representation by means of causal set topology [1] [2] revives interests in topological analysis of the universe. In earlier attempts, topological aspects of the large-scale structures in the universe have been discussed by Hartle and Hawking [4], Ellis and Hawking and others [4]-[6]. Topological aspects of the birth of the universe discussed by Miao Li [7] also lead to a substantial development of mathematical ideas on physical topology of the Universe. The geometry and topology of the universe in the physical realm have been discussed by Lachieze-Rey and Luminet [6] and Mellot [8]. All the care is taken to ensure that fundamental concepts of general topology are not violated. Moreover, the present 
discussion has been developed with a focus on the earlier studies such as Pankaj Joshi [3], Penrose [9] and Vyas [10] wherein causal structure and general topology of the space-time and the global aspects in gravitation and cosmology were discussed rigorously. The computer simulation and animation in $3+1 D$ space in the reference [2] motivates us to further investigate the unexplored aspects of the general topology of space-time.

It is felt that apart from the manifold of the universe, other general topological properties of the universe and the topological changes following them are equally important. The general topology of the universe can be uniquely described if its physical features are specified. A topological space is an abstract conception and thus it is always not possible to attribute physical properties to it. But, the other way round is certainly possible. The topology of a physical object with evident metricity that is inbuilt distance features can certainly be described. Moreover, it is pertinent to mention that the physical realities of the universe [3]-[8] are in perfect agreement with the mathematical conditions discussed in the present paper. Thus, it is good ground to assume that topological considerations could prove to be crucial mathematical constraints on the overall geometry of the universe.

We have several models of the universe at the cross-roads of scientific development, but we consider only one scenario wherein the universe is the single largest entity that encloses every physical object that exists in nature including space and time. The discussion in this paper is developed on two logical grounds such that firstly it justifies the observed facts and secondly it satisfies the mathematical conditions of the whole space topology. The universe without boundary has already been described in the framework of General Theory of Relativity (GTR) too. But, we feel that GTR and Einstein equation describe the universe in a specific physical scenario of a theoretical framework, whereas, general topology of the whole space is absolute truth, and thus it is not limited to any specific physical scenario. This discussion addresses not only the boundary of the universe, but also a detail spatial analysis of the universe with reference to open space, closed space, boundary, compactness, connectedness, limit points, frontier points, interior points, topological transitions and causality and topology. It is concluded in this paper that the general topology of the whole space could be a very good philosophical basis for describing geometry of the universe as it prescribes mathematical constraints on the global geometry of the universe.

\section{Openness, Compactness, and the Boundary}

The metric of the universe can be considered as the super metric or the universal metric defined on the whole space $U$. Then such a universal topology is the set of all possible sets and subsets including the null set. With the consideration that everything that exists in nature is contained in the universe, the complement of the universe is obviously nonexistent.

By definition universal topology being collection of all possible sets and subsets, is always discrete in nature. Thus, the topology of the universe is a discrete topology. Also, the whole space $U$ is always open as well as closed. We explain this geometrical duality using both geometrical physical explanations. Since, $U \cap\{\phi\}=\{\phi\}$; $U$ is closed if its complement $\sim U$ is open and $U$ is open if its complement $\sim U$ is closed, as the null space $\phi$ is both open as well as closed.

Mathematically, if the closure of the space $U$ is given by $\bar{U}$, the condition of the whole space implies that: $U=\bar{U}$.

Hence, if the closure of the universe is equal to the universe itself which is precisely true for the physical universe, the set $U$ of the universe is said to be closed.

An important mathematical corollary [11] asserts that boundary of a space can be given by: $\operatorname{Bd} U=\bar{U}-U$.

But, since $U=\bar{U}$, the boundary of the space vanishes as $\mathrm{Bd} U=\phi$.

Thus, $\mathrm{Bd} U=\phi \Leftrightarrow$ is both open and closed.

This agrees with the conclusion which we have already drawn but by another reason. It is interesting to cite here a mathematical theorem, which states that boundary of a boundary is always empty [11]:

$$
\partial \partial M=\phi .
$$

Furthermore, if $d$ is a metric defined on space $U$, a subset $A$ of $U$ is said to be bounded if there is some number $N$ such that

$$
d\left(a_{1}, a_{2}\right) \leq N \text {; for every pair of points } a_{1}, a_{2} \text { of } A .
$$

If $A$ is bounded, the diameter of $A$ is defined to be the number 


$$
\operatorname{diam}(A)=\text { least upper bound }\left\{d\left(a_{1}, a_{2}\right): a_{1}, a_{2} \in A\right\} .
$$

But, we know that for continuously expanding $U$, particularly in inflationary scenario, this condition cannot be fulfilled as for every pair $a_{1}, a_{2}$; the distance $d\left(a_{1}, a_{2}\right)$ cannot remain less than or equal to a fixed number $N$ forever. The aforesaid conclusion is in agreement with the statement that the universe has no boundary, because a boundary represents in a sense the "edge" of the universe not detected by any astronomical observations [4] [6]. However, the metric of the universe is closed but it cannot be bounded, and therefore it is not at all compact.

\section{Causality and Space-Time Topology}

An event $p$ chronologically precedes another event $q$, denoted by $p \ll q$, if there is a smooth future directed time-like curve from $p$ to $q$ [1]-[3] [9] [10].

If such a curve is non-space-like that is, either time-like or null, we say that $p$ causally precedes $q$, or $p<q$. The chronological future $I^{+}(p)$ of $p$ is the set of all points $q$ such that $p \ll q$. The chronological past of $p$ is defined dually [3]. Thus we have

$$
I^{+}(p)=\{q \in M \mid p \ll q\},
$$

and

$$
I^{-}(p)=\{q \in M \mid q \ll p\} .
$$

The causal future (past) for $p$ can be defined similarly

$$
\begin{aligned}
& J^{+}(p)=\{q \in M \mid p<q\}, \\
& J^{-}(p)=\{q \in M \mid q<p\} .
\end{aligned}
$$

We now observe yet another duality, which can be realized for all $p \ll q \ll r$, such that

$$
I^{-}(r) \supset I^{-}(p),
$$

but,

$$
I^{+}(r) \subset I^{+}(p) .
$$

And similarly, causal future (past) could be defined dually as:

$$
J^{-}(r) \supset J^{-}(p),
$$

and

$$
J^{+}(r) \subset J^{+}(p) .
$$

Proposition 3.1: If there is a smooth future directed time-like curve with all $p \ll q \ll r$ on it, then

$$
I^{-}(r) \supset I^{-}(p) \text { and } I^{+}(r) \subset I^{+}(p) .
$$

Proof: If we consider $p \ll q \ll r$ in the given case, then obviously

$$
q \notin I^{+}(r) \text {, and } q \in I^{+}(p)-I^{+}(r) \text {. }
$$

Thus, the fact that $I^{+}(p)-I^{+}(r) \neq \phi$ implies $I^{+}(r) \subset I^{+}(p)$.

Similarly, one can easily assert that: $q \notin I^{-}(p)$ and $q \in I^{-}(r)-I^{-}(p)$.

And, the fact that $I^{-}(r)-I^{-}(p) \neq \phi$ implies $I^{-}(p) \subset I^{-}(r)$.

Hence is the proposition.

The computer simulation containing animation in $3+1 D$ space in the reference [2] is reassuring that this discussion is on firm footing.

Also, this conveys the feature of open-ended future of the universe at any point of time. If the universe goes on expanding then the topology of the universe will tend to be greater or stronger topology. And the topology of 
the universe at any point of time in future will be stronger or greater than the present topology, and it will further tend to be greater and stronger. This is due to the fact that it will have more and more number of open sets, following this enhancement.

Proposition 3.2: The topology of the present universe is greater or stronger than the topology of the universe in the past and topology of the future universe will be stronger or greater than the present topology of the universe.

Proof: If we consider the topology of the universe defined on the whole space $(U, \mathfrak{I})$, at some point of time in the past then we can construct the topologies of the universe at present and in the future, relative to this. The topology of the present universe can be given as:

$$
\mathfrak{I}_{\text {present }}=\left\{U, \phi, \bigcup\left\{x_{\mu}\right\}\right\} ;
$$

where, $U\left\{x_{\mu}\right\} \subset U$ is the collection of all space-time points accumulated in $U$, after having defined the topology $\mathfrak{I}_{\text {past }}$.

Similarly, we can define the topology of the universe of future as:

$\Im_{\text {future }}=\left\{U, \phi, \cup\left\{x_{v}\right\}\right\}$, where $\bigcup\left\{x_{v}\right\} \subset U$, and $\bigcup\left\{x_{v}\right\}$ is the collection of all space-time points accumulated in $U$, after having defined the topology $\mathfrak{I}_{\text {past }}$; such that $\bigcup\left\{x_{v}\right\} \supset \cup\left\{x_{\mu}\right\}$.

Hence, $\mathfrak{I}_{\text {past }} \subset \mathfrak{I}_{\text {present }} \subset \mathfrak{I}_{\text {future }}$.

In other words the topology of $U$ (past) is smaller or weaker than the topology of $U$ (present), and topology of $U$ (future) will be greater or stronger than the topology of $U$ (present) .

Hence is the proposition.

This further guarantees that the universe will remain unbounded. We can alternatively explain the topological transition of the universe from a weaker or smaller to a stronger or greater topology in terms of matter-energy distribution. Considering the total energy of the universe to be conserved, the continuous expansion of the universe will lead to increase in the intergalactic distances and decline in the matter density over the space. This implies that the topology of the universe in past $U$ (past) was coarser than the $U$ (present), and topology of $U$ (future) will be finer than the $U$ (present) .

\section{Limit Points}

Now, we look for the limit points of the universe. However, this part of the discussion may not be applicable to the consideration of multi-universe Cosmos. If the universe is not static and is continuously expanding, its farthest possible extents can be easily approximated. The radius of the expanding universe can be given by the well-known relation $R=\frac{c}{H}$, or alternatively $R \approx c \tau$; where $H^{-1}$ is Hubble age, $c$ is the velocity of light, and $\tau$ is characteristic time scale or the age of the universe. Alternatively, for the sake of generality, this distance $L_{\text {Horizon }}$ that is (comoving) radius of the observable universe (with or without inflation) is given with a good precision as [6]:

$$
L_{\text {Horizon }} \approx \frac{u}{(1-\gamma)} L_{\text {Hubble }} .
$$

This follows from the logic that the size of the universe can be determined by means of the distance traversed by the fastest moving particle ever since the genesis of the universe. Obviously, this radius is only instantaneous radius and is increasing continuously. Moreover, the universe is considered to be homogeneous and isotropic. With the expansion of the universe, the continuous inclusion of more number of space (or space-time) points restricts the universe from being bounded and compact.

\section{Discussion}

One can define a manifold over a topology if that topological space is locally homeomorphic to $\mathbf{R}^{\mathbf{n}}$, which means it is Hausdorff and possesses metric properties, and connectedness is well described in that. Moreover, a smooth manifold can be described only if continuity as well as differentiability is well defined on it. The manifold of the universe can only be described, if it satisfies these requisite conditions. And we know that it does satisfy them. 
Also, if the origin of the universe is considered to have followed the single event say Big-Bang, then all the physical objects and events in the universe are causally connected. Thus, one can say that the metric of the space-time is connected too. Physically interesting known space-time examples such as the Schwarzschild geometry and Robertson-Walker space-times are topologically Hausdorff. Also, for the sake of the causal regularity of space-time, the continuum of the space-time structure is necessary which would ensure a globally wellbehaved space-time [3]. In nut-shell, at least for the Minkowski space-time, the manifold is globally Euclidean with the topology of $\mathbf{R}^{4}$ over the Lorentzian metric. In case of general theory of relativity, the principles of GTR effectively imply that it is the space-time metric, and the quantities derived from it, that must appear in the equations for physical quantities and that these equations must reduce to the flat space-time case when the metric is Minkowskian. This is the basic content of the GTR where the space-time manifold is allowed to have topologies other than $\mathbf{R}^{4}$ too. However, locally and globally, there could be important differences in the causal structure due to a different local space-time topology, strong gravitational fields and so on [3].

There is a reason to extend the understanding of topology of space-time to the universe at large. Once we understand the nitty-gritty of causal structure and topological aspects of space-time, the understanding of causal structure enables us to deliberate space-like, light-like and time-like events. And the universe at large is nothing but an assembly of space-like, light-like and time-like events. However, the manifold of the universe can have different possible topological features depending upon the physical conditions considered in a specific scenario the global aspects of the general topology of the universe as the whole space remain unchanged. Thus, a prominent suggestion emerges that the mathematical conditionality of the whole space topology can be used as a constraint to describe the overall topology of the universe. In other words, the general topological approach comprises of powerful techniques that could prove to be useful to prescribe mathematical constraints on the global character of the Universe as well as on the manifold of the space-time. In future we can explore frontier points, interior points and other topological notions too.

\section{Acknowledgements}

The author is grateful to Professor T. Pandmanabhan of Inter University Centre for Astronomy and Astrophysics (IUCAA) for his encouragement and patient reading of the manuscript.

\section{References}

[1] Aalok, P. (2012) Null Cone Representation of Interactions and Causal Structure. Physics Essays, 25, 228-232. http://dx.doi.org/10.4006/0836-1398-25.2.228

[2] Aalok, P. (2013) Computational Investigations of the Null Cone Representation of Interactions. Physics Essays, 26, 24-26. http://dx.doi.org/10.4006/0836-1398-26.1.24

[3] Joshi, P.S. (1993) Global Aspects in Gravitation and Cosmology. Oxford Science Publications, Oxford.

[4] Hartle, J.B. and Hawking, S.W. (1983) Wave Function of the Universe. Physical Review D, 28, 2960-2975; Hawking, S.W. (1984) Quantum State of the Universe. Nuclear Physics B, 39, 257-276.

[5] Hawking, S.W. and Ellis, G.F.R. (1973) The Large Scale Structure of Space-Time. Cambridge University Press, Cambridge.

[6] Lachieze-Rey, M. and Luminet, J.P. (1995) Cosmic Topology. Physics Reports, 254, 135-214. http://dx.doi.org/10.1016/0370-1573(94)00085-H

[7] Li, M. (1986) On Topological Aspects of the Birth of the Universe. Physics Letters B, 173, 229-232. http://dx.doi.org/10.1016/0370-2693(86)90507-1

[8] Melott, A.L. (1990) The Topology of the Large Scale Structure in the Universe. Physics Reports, 193, 1-39. http://dx.doi.org/10.1016/0370-1573(90)90162-U

[9] Penrose, R. (1972) Techniques of Differential Topology in Relativity. A. M. S. Colloquium Publications, SIAM Philadelphia. http://dx.doi.org/10.1137/1.9781611970609

[10] Vyas, U.D. and Joshi, P.S. (1989) Topological Techniques in General Relativity in Geometry and Topology. World Scientific, Singapore, 315-331.

[11] Munkres, J.R. (1975) Topology: A First Course. Prentice-Hall Inc., Englewood Cliffs. 
Scientific Research Publishing (SCIRP) is one of the largest Open Access journal publishers. It is currently publishing more than 200 open access, online, peer-reviewed journals covering a wide range of academic disciplines. SCIRP serves the worldwide academic communities and contributes to the progress and application of science with its publication.

Other selected journals from SCIRP are listed as below. Submit your manuscript to us via either submit@scirp.org or Online Submission Portal.
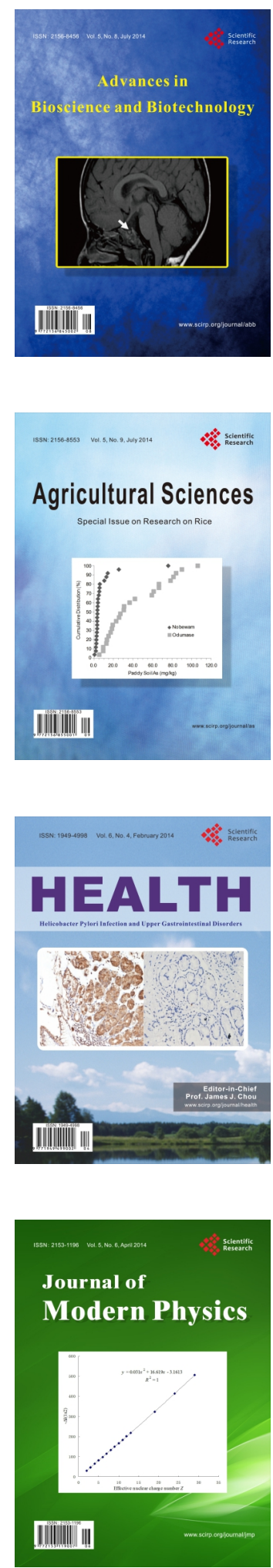
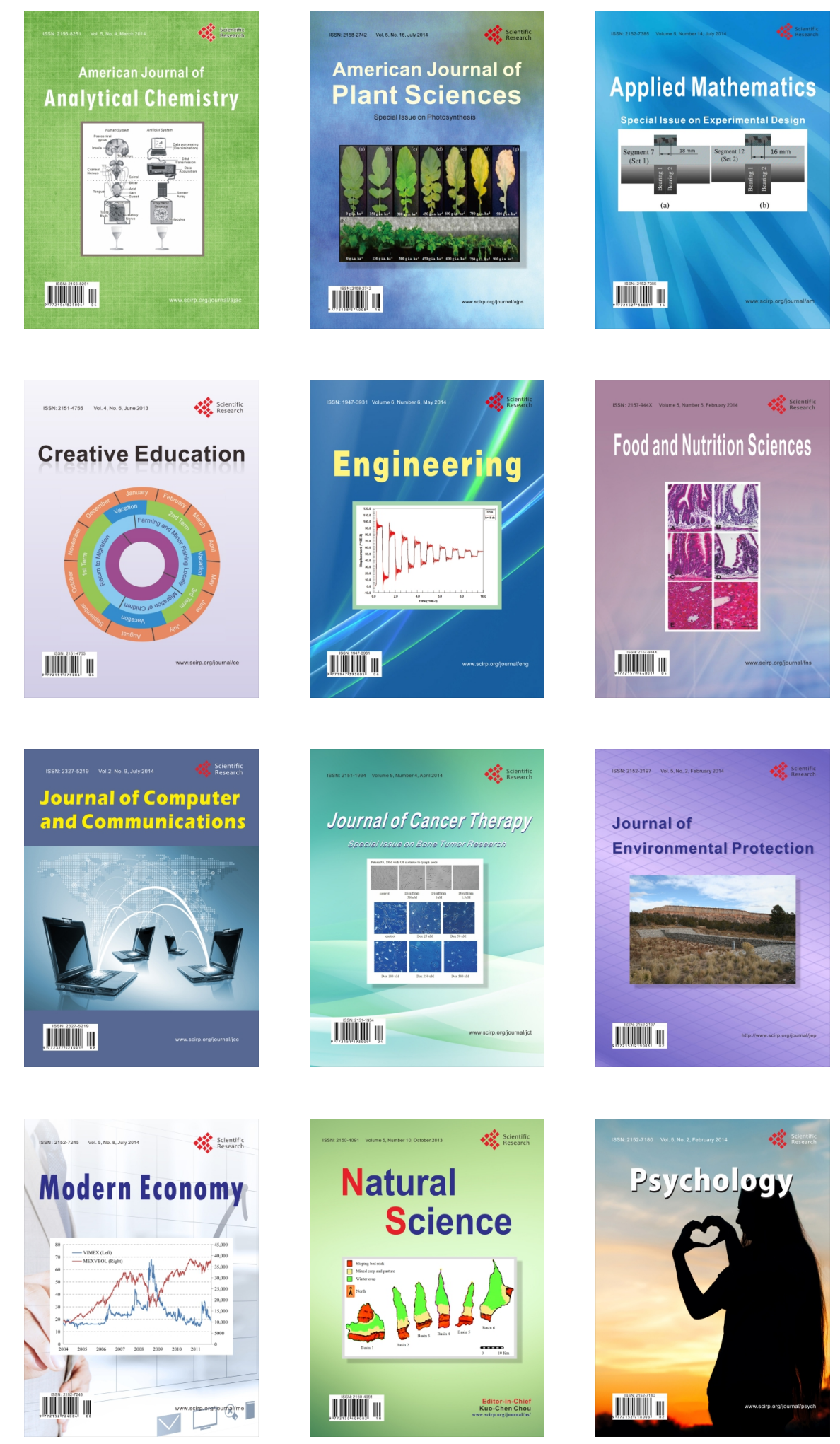